\title{
Gene therapy of cardiac failure
}

\author{
M.I. Qadir ${ }^{1}$, I. Munir ${ }^{2}$, T.U. Nisa ${ }^{3}$ \\ ${ }^{1-3}$ College of Pharmacy, GC University, Faisalabad, Pakistan
}

\begin{abstract}
Every year in the world there is increasing death rates due to heart failure. So it is very essential to cure the heart failure on a long term basis. For this purpose, gene therapy is somehow the best way. We can do this by discovering new pharmacological targets, producing regenerative medicine by use of stem cells, using adipose tissues for cardiac repair, using resynchronization therapy by changing the gene expressions, by correcting the gene expression of connexin43, by enhancing the mitochondrial function, insertion of ACE gene, curing the down regulation of calcium signaling protein, use of micro RNAs, modification of cardiovascular ion channels by gene therapy, silencing the myotrophin gene. Heart failure means the loss of heart functions; heart stops its working which may lead to death. Now a day, heart failure is dominating in the world. There are lots of factors involve in this disease.
\end{abstract}

Key words: Cardiac failure, gene therapy, stem cells, gene expressions, ACE gene.

\section{Gene therapy for heart failure}

Gene therapy is somehow the best way to cure heart failure. There has been much improvement in this field in the last 10 years and it has shown very successful results. By using this, we can cure many basic defects in the heart, functioning at the cellular level. Multiple patho-physiological mechanisms can also be cured by using gene therapy such calcium fluxes during excitation of heart can be enhanced. And nowadays further researches are going on. ${ }^{1}$ Cardiac system is very important in the human health. We have studied the cardiac rhythmicity, its physiology and pathogenesis, the cure of heart failure and cardiac hypertrophy. When there is change in the rhythmicity of the heart, the change in gene expression is going on too. This is involved in Correspondence: M.I. Qadir E-mail:mrimranqadir@hotmail.com causing large number of heart diseases. We can cure these diseases if we completely get knowledge about these gene expressions. ${ }^{2}$ Gene therapy plays an important role in curing the heart diseases of genetic disorders and acquired pathophysiologies such as arteriosclerosis and heart failure. But there is not too much progress in this field because of lack of gene delivery systems for localizing gene therapy to specific site to optimize transgene expression and efficacy. ${ }^{3}$ Cardiac remodeling and improvement its function can be brought about by using ILK gene therapy which can also increase the angiogensis, increase cardiomyocyte and reduce apoptosis after myocardial infarction. This may also be helpful in the postinfarct remodeling and subsequent heart failure. ${ }^{4}$ In many cardiovascular diseases there are abnormalities associated with the expression or function of ion 
Journal of College of Medical Sciences-Nepal,2010,Vol-6,No-4

channels. So, for its cure small molecule drugs can be used for the delivery of gene to modify the ion channels. Basically we can cure the hypertrophy, heart failure and hypertension by using the gene therapy. For the delivery of gene the use of viral vectors is under study. ${ }^{5}$

\section{Gene and cell therapy for heart failure}

Cardiac cell and gene therapy have entered into clinical trials for the patients of chronic congestive heart failure. By using gene therapy we can increase the Calcium pump in the sarcoplasmic reticulum and hence can improve the contractility of the heart. In case of cell therapy its clinical benefits are not clear. However, cell therapy by use of myoblasts is somehow beneficial in case of myocardial infarction. ${ }^{6}$

\section{Pharmacoepigenetics in heart Failure}

Epigenetic changes play an important role in the variation of predisposition of diseases and recent evidences of epigenetic changes also play an important role in heart failure and cardiac hypertrophy. These epigenetic changes include the histone modification, DNA methylation and micro RNAs. ${ }^{7}$ The vascular endothelial growth factors are implicated in the pathogenesis of the coronary artery disease and acute myocardial infarction. It is reported that there are few SNPs which are associated with the variations in serum concentrations of VEGF and susceptibility to CAD and acute coronary syndrome. VEGF gene delivery can be used for treating the CAD but it does not show full efficacy. ${ }^{8}$ Corin is a allele which shows its expressions in the cardiomyocytes If there occur any mutation in this allele it may lead to hypertension and systolic heart failure. On experimental basis and pharmacogenomic interactions in blacks it is concluded that any mutation in this allele leads to heart failure. ${ }^{9}$ There is a considerable morbidity and mortality rate due to the heart failure. There are some factors still unknown such as-inflammatory mechanisms contribute in heart failure. So HMGB1 is the key danger signal that is released by the necrotic cells and this signal is given by the RAGE. HMGB1 and RAGE are the key components involved in inflammatory cardiomyopathy eventually leading to heart failure. ${ }^{10}$ When there are changes in gene expression of connexin 43 protein involved in normal heart functioning, they become crucial factors in normal cardiac development and its functions. Several heart related diseases can occur such as heart failure, atherosclerosis etc. But recent studies have developed the potential therapies related to connexin 43 to cure the cardiovascular diseases and signaling pathways. ${ }^{11}$

\section{Developmental and genetic aspects of atrial fibrillation}

Atrial fibrillation is a common form of cardiac arrhythmia. Abnormal rhythm may cause shortness of breath, heart failure and other heart related diseases. It has been estimated that there is $85 \%$ chance of development of atrial fibrillation in an individual with at least one parent with the history of atrial fibrillation. Recent studies have shown that genetic factor is also involved in $\mathrm{AF}^{12}$

\section{Types of gene therapy}

Gene therapy can be done by several methods but the usually ones are gene targeting, changing gene expressions and gene silencing.

Pharmacogenetic effects of ACE inhibitors on heart failure 


\section{M.I. Qadir et al, Gene therapy of cardiac failure}

ACE inhibitors have very good effects on patients who suffered from diastolic heart failure. It has been shown by experiments that $\mathrm{ACE}$ gene insertion in diastolic heart failure patients can decrease the long term mortality rate as compared to the use of other drugs. ${ }^{13}$ The failing heart becomes depleted of energy and it is involved in the progression of disease. Mitochondrial function becomes depressed which can lead to chronic heart failure. In chronic heart failure, the patients which were treated with the ACE inhibitors had more cardiac oxidative capacity and were partially able to protect cardiac mitochondrial function and transcription cascade than the patients who were receiving the endothelin-1 and angiotensin II. ${ }^{14}$

\section{Stem cells and adipose tissue as regenerative medicine for myocardial infarction}

Regenerative therapies have shown possible ways to cure severe heart failure which is due to myocardial infarction and ischemic cardiomyopathy. For this purpose we use several potential cell sources such as bone marrow stem cells, skeletal myocytes and embryonic stem cells. ES cells are used because they are highly proliferative. They are used for mass production. So, we have formed the IPS cells by the gene transfer of 4 transcription factors into the IS cells. IPS cells can be differentiated into cardiomyocytes same as ES cells and they have become ideal cell source of regenerative medicine. ${ }^{15}$ Bone marrow cells such as stem cells and progenitor cells can be used to enhance the functional recovery after acute myocardial infarction. As these cells are highly proliferative, they can easily differentiate into cardiomyocytes. There are difficulties with them in some situations. Recently, it has been discovered that the adipose tissue contains adult endothelial proginator cells and multipotent mesenchymal stem cells. They are also highly proliferative and can easily differentiate into cardiomyocytes. The similarity between stem cells and adipose tissues has shown that adipose tissues can be used an alternative, and also as a source for repairing damaged tissues in infarcted heart. ${ }^{16}$ In developed countries, myocardial infarction is one of the causes of mortality. So, bone marrow mesenchymal stem cells injection is given and this therapy has the ability to regenerate the cardiomyocytes in heart failure. ${ }^{17}$

\section{New pharmacological targets: aldosterone receptor as an example}

Heart failure has multiple etiologies. There are numerous therapeutic targets such as the neurohormonal blockage e.g. adrenergic and renin angiotensin aldosterone system. For better identification of signal pathways we use animal models classical or genetically modified. Although the patho-physiological and physiological patterns are different in animals than the humans, it provides us the new hypothesis and therapeutic approaches. Gene targeting have open new discoveries in pharmacological targets in heart failures, such as in case of aldosterone and its receptors. Mineralocorticosteroids are involved in the pathophysiology of heart failure and by using the new therapeutic targets of aldosterone, we have made many improvements in heart failure. ${ }^{18}$

\section{Beta-adrenergic blockade attenuates cardiac dysfunction}

Beta-adrenoceptor blockade is the useful therapy for congestive cardiac failure. In laboratory three weeks 
Journal of College of Medical Sciences-Nepal,2010,Vol-6,No-4

after inducing myocardial infarction rats were treated with selective beta- 1 blocker atenolol and nonselective beta blocker propranolol and it showed very good results by improving heart function ability. These blockers attenuated the MI-induced depressions in myofibrillar $\mathrm{Ca}(2+)$ stimulated ATPase activity and phosphorylated cTnl protein content. Hence it is thought that the improvement of cardiac function by using beta blockers is associated with the attenuation of myofibrillar remodeling. ${ }^{19}$ Beta1-adnergic receptor Arg389Gly and beta2-adnergic receptor Arg16Gly single neucleotide gene polymorphisms are not related to the response to the carvedilol therapy. But the Gln27Glu single neucleotide gene polymorphism is a determinant of the left ventricular ejection fraction response to this agent in chronic heart failure patients. ${ }^{20}$

\section{Cardiac resynchronization therapy}

Electromechanical dyssynchrony affects the regional cardiac transcriptome causing gene expression in the anterior LV wall. Cardiac resynchronization can correct the gene expression in the anterior wall of LV. Like this CRT can improve the heart function by working at the genomic level. ${ }^{21}$ By taking tissues from failing hearts it has been shown that there is down regulation of $\mathrm{K}^{+}$ currents and there is alteration in depolarizing $\mathrm{Na}^{+}$and $\mathrm{Ca}^{++}$currents, that's why, there is alteration in the electrical signaling and hence slowing down the conduction. For this purposes we use the cardiac resynchronization therapy for electrical remodeling in failing heart. $^{22}$

\section{Cardiac-targeted delivery of regulatory RNA molecules}

Nowadays, RNA has become fully understood and it can be used as a tool in medicine and molecular biology. As a therapeutic use, RNA interference can be used to silencing of specific genes and microRNA modulation can be used to alter complex gene expression patterns. It can be used to cure heart failure but still, we are unable to find the target sides. Within next decade RNA based therapy will be used for cardiovascular therapy. ${ }^{23}$ MicroRNAs have recently emerged and play a central role in the gene expression. And it has been studied that they can be implicated in every pathological process of cardiovascular systems such as cardiac hypertrophy, heart failure, cardiac fibrosis and vascular atherosclerosis. By forcefull expression or suppression of the single microRNA we can cause many pathological alterations. ${ }^{24}$ By using the RNA interference we can stop or silence the myotrophin gene which further prevents the NFkappaB activation, whose activation is associated with the cardiac hypertrophy. This method becomes the excellent therapeutic means for the treatment of heart failure. ${ }^{25}$

\section{Down regulation of $\mathrm{Ca}^{2+}$ signaling proteins in cardiac hypertrophy}

Calcium signaling has very important role in heart functioning. If there is prolonged rise of calcium in the cytosol, it may result in cardiac hypertrophy and the high level is due to calcium signaling proteins. These proteins are down regulated in cardiac hypertrophy and this imbalance also leads to cardiac failure. Gene transfer experiments are going on in this way which will be helpful in gene therapy of cardiac failure. ${ }^{26}$ Gene transfer of sarco-endoplasmic reticulum ATPase in the rat models with chronic heart failure had shown many 


\section{M.I. Qadir et al, Gene therapy of cardiac failure}

improvements in the past. Now, this gene (SERCA2a) transfer has also been done in big animals such as beagls with chronic heart failure and its results show significant improvement in heart function by increasing the SERCA2a expression and also by improving the left ventricular systolic function. ${ }^{27}$ Sarco/endoplasmic reticulum plays an important role in contraction of heart, as the SERCA2a expression plays an important role in regulation of calcium. Change in the expression of SERCA2a leads to the low levels of calcium and ultimately leads to heart failure. So the trails are going on to restore the SERCA2a expressions by using adeno-associated virus type $1 .^{28}$

\section{Targeting transcriptional pathway in cardiomyocytes by curcumin}

In industrialized countries there is more mortality rate because of hypertension and post myocardial infarction heart failure. Actually cardiomyocytes undergo maladaptive hypertrophy which influences the gene expression. Excessive acetylation of cardiomyocyte nuclei is responsible for the maladaptive cardiomyocyte hypertrophy. Curcumin can inhibit the nuclear acetylation and is useful in treating the heart failure. ${ }^{29}$

\section{Prolyl hydroxylase inhibition attenuates post- ischemic cardiac injury}

Ischemia/reperfusion affects the endoplasmic reticulum and initiate the unfolded protein response. However, it fails to release stress of ER which leads to apoptosis causing post ischemic cardiac injury. It has been studied that propyl hydroxylase inhibition can attenuate the post ischemic cardiac injury by reducing the apoptosis and inducing protective ER stress protein and by decreasing the pro-apoptotic components of UPR. ${ }^{30}$

\section{Conclusion}

In conclusion, that gene therapy is the best way to cure heart failure. By using the different techniques of gene therapy we can obtain long lasting cure regarding the heart failure. Still more studies and experiments are going on in this field and it is expected that it will be more helpful in the future and also for the cure of other diseases.

\section{References}

1. J.L. Reyes-Juárez, A. Zarain-Herzberg. Gene therapy for heart failure. Arch Cardiol Mex. 2009;79(2):147-56.

2. M.J. Sole, T.A. Martino. Diurnal physiology: core principles with application to the pathogenesis, diagnosis, prevention, and treatment of myocardial hypertrophy and failure. J Appl Physiol. 2009;107(4): 1318-27.

3. I. Fishbein, M. Chorny, R.J. Levy. Site-specific gene therapy for cardiovascular disease. Curr Opin Drug Discov Devel. 2010;13(2):203-13.

4. L. Ding, X. Chen, L. Zhang et al. Increased expression of integrin-linked kinase attenuates left ventricular remodeling and improves cardiac function after myocardial infarction. Circulation. 2009;120(9):764-73.

5. S. Telemaque, J.D. Marsh. Modification of cardiovascular ion channels by gene therapy. Expert Rev Cardiovasc Ther. 2009;7(8):939-53.

6. E.D. Muinck . Gene and cell therapy for heart failure. Antioxid Redox Signal. 2009;11(8):2025-42. 
7. I.M. Leach, P. van der Harst, de Boer RA. Pharmacoepigenetics in Heart Failure. Curr Heart Fail Rep.2010;7(2):83-90.

8. D. Petrovic. The role of vascular endothelial growth factor gene as the genetic marker of atherothrombotic disorders and in the gene therapy of coronary artery disease. Cardiovasc Hematol Agents Med Chem. 2010; 8(1):47-54.

9. J.E. Rame, S.W. Tam, D. McNamara, et al. Dysfunctional corin allele is associated with impaired brain natriuretic peptide processing and adverse outcomes in blacks with systolic heart failure: results from the Genetic Risk Assessment in Heart Failure substudy. Circ Heart Fail. 2009;2(6):541-8.

10. H.C. Volz, Z. Kaya, H.A. Katus, et al. The role of HMGB1/ RAGE in inflammatory cardiomyopathy. Semin Thromb Hemost. 2010;36(2):185-94.

11. Y.N. Song, H. Zhang, J.Y. Zhao et al. Connexin 43, a new therapeutic target for cardiovascular diseases. Pharmazie. 2009; 64 (5):291-5.

12. A.V. Postma, L.R. Dekker, A.T. Soufan et al. Developmental and genetic aspects of atrial fibrillation. Trends Cardiovasc Med. 2009;19(4):123-30.

13. C.K. Wu, J.L. Luo, C.T. Tsai, et al. Demonstrating the pharmacogenetic effects of angiotensin-converting enzyme inhibitors on long-term prognosis of diastolic heart failure. Pharmacogenomics J. 2010;10(1):46-53.

14. A. Garnier, J. Zoll, D. Fortin, et al. Control by circulating factors of mitochondrial function and transcription cascade in heart failure: a role for endothelin-1 and angiotensin II. Circ Heart Fail. 2009;2(4):342-50.
15. T. Egashira, K. Fukuda. The possibility of regenerative medicine for myocardial infarction and ischemic cardiomyopathy by using several stem cells. Nippon Rinsho. 2010; 68(4):731-6.

16. R. Madonna, R. De Caterina. Adipose tissue: a new source for cardiovascular repair. J Cardiovasc Med (Hagerstown). 2010;11(2):71-80.

17. O. Martínez de Ilárduya, J. Barallobre Barreiro, I. Moscoso et al. Gene expression profiles in a porcine model of infarction: differential expression after intracoronary injection of heterologous bone marrow mesenchymal cells. Transplant Proc. 2009;41(6):2276-8.

18. C. Latouche, F. Jaisser. Contribution of transgenesis models in discovery of new pharmacological targets in heart failure: aldosterone receptor as an example. Therapie. 2009;64(2):81-6.

19. J. Machackova, S.K. Sanganalmath, V. Elimban et al. beta-adrenergic blockade attenuates cardiac dysfunction and myofibrillar remodeling in congestive heart failure. J Cell Mol Med. 2010.

20. M. Metra, L. Covolo, N. Pezzali et al. Role of betaadrenergic receptor gene polymorphisms in the longterm effects of beta-blockade with carvedilol in patients with chronic heart failure. Cardiovasc Drugs Ther. 2010;24(1):49-60.

21. A.S. Barth, T. Aiba, V. Halperin et al. Cardiac resynchronization therapy corrects dyssynchronyinduced regional gene expression changes on a genomic level. Circ Cardiovasc Genet. 2009;2(4):371-8.

22. T. Aiba, G.F. Tomaselli. Electrical remodeling in the failing heart. Curr Opin Cardiol. 2010;25(1):29-36. 
M.I. Qadir et al, Gene therapy of cardiac failure

23. W. Poller, R. Hajjar, H.P. Schultheiss et al. Cardiactargeted delivery of regulatory RNA molecules and genes for the treatment of heart failure. Cardiovasc Res. 2010;86(3):353-64.

24. Z.W. Pan, Y.J. Lu, B.F. Yang. MicroRNAs: a novel class of potential therapeutic targets for cardiovascular diseases. Acta Pharmacol Sin. 2010;31(1):1-9.

25. S. Gupta, R. Maitra, D. Young. Silencing the myotrophin gene by RNA interference leads to the regression of cardiac hypertrophy. Am J Physiol Heart Circ Physiol. 2009;297(2):H627-36.

26. A.M. Prasad, G. Inesi. Downregulation of $\mathrm{Ca} 2+$ signalling proteins in cardiac hypertrophy. Minerva Cardioangiol. 2010;58(2):193-204.
27. Y.F. Mi, X.Y. Li, L.J. Tang. Improvement in cardiac function after sarcoplasmic reticulum $\mathrm{Ca} 2+-\mathrm{ATPase}$ gene transfer in a beagle heart failure model. Chin Med $J$ (Engl). 2009;122(12):1423-8.

28. L. Lipskaia, E.R. Chemaly, L. Hadri et al. Sarcoplasmic reticulum $\mathrm{Ca}(2+)$ ATPase as a therapeutic target for heart failure. Expert Opin Biol Ther. 2010;10 (1):29-41.

29. T. Morimoto, Y. Sunagawa, M. Fujita et al. Novel Heart Failure Therapy Targeting Transcriptional Pathway in Cardiomyocytes by a Natural Compound, Curcumin. Circ J. 2010.

30. R. Natarajan, F.N. Salloum, B.J. Fisher et al. Prolyl hydroxylase inhibition attenuates post-ischemic cardiac injury via induction of endoplasmic reticulum stress genes. Vascul Pharmacol. 2009;51(2-3):110-8. 